\title{
Accuracy of stroke volume variation and pulse pressure variation to predict fluid responsiveness in patients with thoracic kyphosis
}

\author{
Yu Chen ${ }^{1 \#}$, Xiaoming Guo ${ }^{2 \#}$, Jiannan Fu ${ }^{1 \#}$, Tong Dong ${ }^{3}$, Xiaomei Liu ${ }^{1}$, Hao Lv $^{1}$ \\ ${ }^{1}$ Department of Anesthesiology, The Sixth Medical Center of Chinese PLA General Hospital, Beijing, China; ${ }^{2}$ Department of Anesthesiology, The \\ 900th Hospital of Joint Logistics Support Force, Fuzhou, China; ${ }^{3}$ Department of Obstetrics and Gynecology, The Sixth Medical Center of Chinese \\ PLA General Hospital, Beijing, China \\ Contributions: (I) Conception and design: All authors; (II) Administrative support: None; (III) Provision of study materials or patients: None; (IV) \\ Collection and assembly of data: Y Chen, X Guo; (V) Data analysis and interpretation: Y Chen, J Fu; (VI) Manuscript writing: All authors; (VII) \\ Final approval of manuscript: All authors. \\ \#These authors contributed equally to this work. \\ Correspondence to: Hao Lv. Department of Anesthesiology, The Sixth Medical Center of Chinese PLA General Hospital, Beijing, China. \\ Email: mzk6plagh@163.com.
}

Background: This study aims to evaluate the ability of stroke volume variation (SVV) and pulse pressure variation (PPV) to predict fluid responsiveness in mechanically ventilated patients with thoracic kyphosis.

Methods: A total of 35 patients diagnosed with thoracic kyphosis undergoing corrective surgery were studied. For all patients, the Vigileo/FloTrac system was used for analysis. Hemodynamic data such as mean arterial pressure (MAP), heart rate (HR), stroke volume (SV), stroke volume index (SVI), cardiac output (CO), cardiac output index (CI), SVV, and PPV were recorded before and after volume expansion (VE). Fluid responsiveness was defined as an increase in SVI $\geq 10 \%(\Delta \mathrm{SVI} \geq 10 \%)$. Patients were divided into responders and non-responders as determined by changes in $\Delta$ SVI $\geq 10 \%$ and $<10 \%$. Nonparametric Wilcoxon rank sum test was used to compare the hemodynamic parameters of Responders and Non-responders before and after VE. Pearson correlation analysis was used to analyze the values of SVV, PPV and $\triangle$ SVI. The receiver operating characteristic (ROC) curve of each hemodynamic index was drawn to determine its accuracy and threshold.

Results: Two patients were excluded. There was no significant difference in patients' characteristics between Responders and Non-responders. After VE, there were no significant changes in HR, MAP, and $\mathrm{SV}$ in both responders and non-responders, but CI were significantly changed in the two groups. SVI and $\mathrm{CO}$ increased significantly in responders before and after VE, but not in non-responders. VE also caused decreases of PPV and SVV in both responders and non-responders. Before VE, the SVV and PPV correlated with $\triangle$ SVI in responders $(\mathrm{r}=0.621, \mathrm{r}=0.569$, respectively, $\mathrm{P}<0.05)$, but neither the SVV nor PPV correlated with $\triangle \mathrm{SVI}$ in non-responders $(\mathrm{P}>0.05)$. The areas under the ROC curves of patients with thoracic kyphosis were 0.872 (95\% CI: 0.719-1.000) for SVV and 0.833 (95\% CI: 0.667-1.000) for PPV. The threshold of the SVV of patients with thoracic kyphosis was $13.5 \%$, and the threshold of PPV was $14.5 \%$.

Conclusions: Both SVV and PPV can be used as effective indictors to monitor volume changes in patients with thoracic kyphosis.

Keywords: Stroke volume variation (SVV); pulse pressure variation (PPV); thoracic kyphosis

Submitted Apr 10, 2021. Accepted for publication Jun 02, 2021.

doi: 10.21037/apm-21-1211

View this article at: https://dx.doi.org/10.21037/apm-21-1211 


\section{Introduction}

With the development of spinal surgery, more and more kyphosis surgery is carried out. It is crucial to maintain critical organ perfusion and hemodynamic stability during surgery, as this operation is often prone to hemorrhage and a long operation time $(1,2)$. In patients with thoracic kyphosis, chest deformity may seriously affect the patient's heart and lung function, bringing more challenges to the intraoperative anesthetic management, especially in volume management.

Commonly used static hemodynamic parameters, such as central venous pressure (CVP) and pulmonary artery wedge pressure (PAWP), are easily affected by right ventricular function and body position among other factors, and are often not capable of predicting a cardiac response to fluid therapy (3). Conversely, stroke volume variation (SVV) and other dynamic hemodynamic parameters, which are based on the interaction of the heart and lungs during mechanical ventilation, have been proven to be good indexes for predicting fluid volume in patients undergoing neurosurgical procedures, cardiac surgery, liver transplantation, and laparoscopy-assisted surgery (4-7).

To our knowledge, there are few articles on SVV and pulse pressure variation (PPV) in the prediction of intraoperative volume monitoring in patients with thoracic kyphosis. It is unclear whether chest deformity affects the accuracy of SVV and PPV, and whether it can cause changes in their threshold values. These questions will be explored and discussed below. We present the following article in accordance with the STARD reporting checklist (available at https://dx.doi.org/10.21037/apm-21-1211).

\section{Methods}

\section{Patients and anesthesia}

This prospective study was conducted at the outpatient clinic of the Chinese PLA general hospital between January and November in 2020. This study followed the tenets of the Declaration of Helsinki (as revised in 2013). The whole protocol was approved by the institutional review board committee of PLA General Hospital in China and written informed consent was obtained from each participant after the nature and possible results of the study were explained (Trial registration number: S2020-006-02). Mechanically ventilated ASA I-II patients $(\mathrm{n}=35)$ with thoracic kyphosis were included in this trial, and were arranged to undergo surgical treatment of selective thoracic kyphosis correction.
All patients were normal in the Allen test. The age of patients was from 15 to 47 , and the maximum Cobb's angle was 35 to 60 degrees. Patients with hypertension, coronary heart disease, arrhythmia, left ventricular ejection fraction (LVEF) $<50 \%$, congenital cardiovascular malformation, and peripheral vascular disease were excluded, as well as patients with a long-term history of oral vasoactive drugs and patients who used vasoactive drugs before and during liquid treatment. The flow chart of the study is shown in Figure 1.

All patients were given $0.5 \mathrm{mg}$ atropine intramuscularly 30-40 min before entering the operating room. After the patient arrived in the operating room, routine monitoring including non-invasive blood pressure, electrocardiogram, and pulse oximetry was applied. Adequate topical anesthesia in the mouth and throat was given concurrently. After midazolam $(2 \mathrm{mg})$ and sufentanil $(10 \mu \mathrm{g})$ were administered, cricothyroid membrane puncture was performed. After the puncture needle entered into the trachea, $40 \mathrm{mg}$ tetracaine was intratracheally injected, and 5 min later, tracheal intubation was performed through the mouth with a fiberoptic bronchoscope. After successful intubation, propofol (2-3 mg/kg, i.v.), sufentanil (0.5-0.6 $\mu \mathrm{g} / \mathrm{kg}$, i.v.), and rocuronium $(0.6-0.9 \mathrm{mg} / \mathrm{kg}$, i.v.) were administered, and then the anesthesia machine was connected for mechanical ventilation in the volume control mode with a tidal volume of $8 \mathrm{~mL} / \mathrm{kg}$, an inspired oxygen concentration of $40-60 \%$, and an inspiratory/expiratory ratio of 0.5 . The respiratory rate was adjusted to maintain an end tidal carbon dioxide between 35 and $40 \mathrm{mmHg}$. Patients were maintained with propofol (100-200 mg/h, i.v.), desflurane (4-6\%, inhalation), and rocuronium (20 mg intermittently, i.v.). Then, an arterial catheter (REFRA-04220, Arrow International Inc., Reading, PA, USA) was inserted in the left radial artery and a central venous catheter (Certofix Duo V720, Braun International Inc., Melsungen, Germany) was placed through the right femoral vein. The pressure sensor was horizontally zeroed at the midline of the axillary line. The bispectral index (BIS, Aspect $1000^{\mathrm{TM}}$, Aspect Medical Systems Inc., Natick, MA, USA) was monitored between 30 and 40 during the whole operation.

\section{Hemodynamic monitoring}

A dedicated transducer (FloTrac ${ }^{\mathrm{TM}}$, Edwards Lifesciences, LLC, Irvine, CA, USA) was connected to the radial arterial line on one side and to the Vigileo System (Vigileo ${ }^{\text {TM }}$ Edwards Lifesciences, LLC, Irvine, CA, USA) on the other side. It can continuously monitor cardiac output 


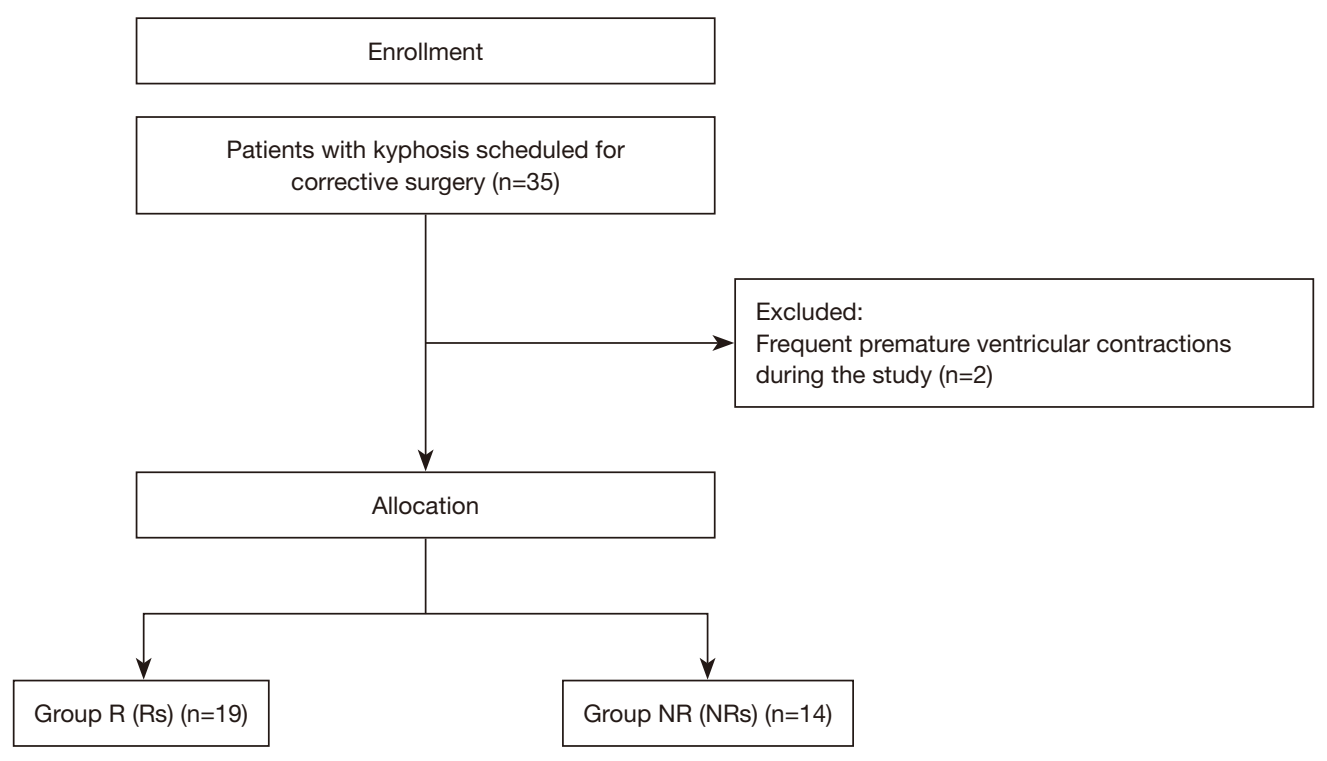

Figure 1 The flow chart of the study. Rs, responders; NRs, non-responders.

(CO), cardiac output index (CI), stroke volume (SV), stroke volume index (SVI), and SVV. SV is calculated as $\mathrm{K} \times$ pulsation, where pulsation is the standard deviation of arterial pressure every 20 seconds, and $\mathrm{K}$ is the factor of arterial compliance and vascular resistance. SVV is the percentage change of $\mathrm{SV}$ in mechanical ventilation, and its calculation formula is $\mathrm{SVV}(\%)=\left(\mathrm{SV}_{\text {max }}-\mathrm{SV}_{\text {min }}\right) / \mathrm{SV}_{\text {mean }}$, in which the maximum and minimum $\mathrm{SV}$ are the average values of the $4 \mathrm{SV}$ extremums in 20 seconds, and the mean $\mathrm{SV}$ is the average value in that period.

The calculation method of PPV is similar, and it is calculated according to the change value of pulse pressure $(\mathrm{PP})$, whose calculation formula is $\mathrm{PPV}(\%)=\left(\mathrm{PP}_{\max }-\right.$ $\left.\mathrm{PP}_{\text {min }}\right) / \mathrm{PP}_{\text {mean }}$. The maximum and minimum $\mathrm{PP}$ are the average values of the 4 extreme values of $\mathrm{PP}$, and the mean $\mathrm{PP}$ is the average value of $\mathrm{PP}$ in this period. SVI is recorded by the system, which is used to distinguish responders and non-responders after volume expansion (VE). During the trial, the following items were recorded simultaneously from the system: mean arterial pressure (MAP), heart rate (HR), CO, CI, SV, SVI, SVV, and PPV.

\section{Study protocol}

This study evaluated the capacity of SVV and PPV to predict fluid responsiveness in patients with thoracic kyphosis during surgery. The VE was started after anesthesia induction and before the operation, beginning in the prone position. During the study, the values of MAP, HR, CO, CI, SV, SVI, SVV, and PPV were recorded before (T0) and after (T1) fluid loading. A hydroxyethyl starch solution (6\%, 130/0.4, Fresenius Kabi, Germany) was infused between 10-15 min for fluid therapy. Hemodynamic measurements were performed before and within $30 \mathrm{~s}$ after VE without stimulation. All patients were studied before the operation began and the patients were in the prone position. During the VE, ventilator settings were kept consistent.

\section{Statistical analysis}

All hemodynamic data were expressed as mean values \pm SD. The patients were divided into responders and nonresponders according to $\Delta S V I \geq 10 \%$ and $<10 \%$ before and after VE. The nonparametric Wilcoxon rank-sum test was used to compare the hemodynamic parameters of responders and non-responders before and after VE. Pearson correlation analysis was used to analyze the values of SVV and PPV, and the receiver operating characteristic (ROC) curve of each hemodynamic index was generated. According to the area under the curve, the predictive ability and the diagnostic thresholds of SVV and PPV were determined. A P value less than 0.05 was regarded as statistically significant. All statistical data were analyzed by the software SPSS 22.0 (SPSS Inc., Chicago, IL, USA). 
Table 1 Patients characteristics between the two groups

\begin{tabular}{lcc}
\hline Items & Responders $(\mathrm{n}=19)$ & Non-responders $(\mathrm{n}=14)$ \\
\hline Age $(\mathrm{yr})$ & $30.4 \pm 2.1$ & $28.5 \pm 3.6$ \\
Gender $(\mathrm{M} / \mathrm{F})$ & $17 / 2$ & $14 / 1$ \\
Height $(\mathrm{cm})$ & $164.3 \pm 7.2$ & $166.9 \pm 6.7$ \\
Weight (kg) & $70.4 \pm 4.1$ & $67.5 \pm 6.6$ \\
Max Cobb's angle (degree) & $45.8 \pm 5.7$ & $50.2 \pm 6.1$ \\
Operation time (min) & $275.1 \pm 29.3$ & $296.4 \pm 32.5$ \\
\hline
\end{tabular}

Data are presented as means \pm SDs or the number. Responders were patients whose $\Delta S V I$ increased $\geq 10 \%$ after fluid loading, while nonresponders were patients whose $\Delta S V I$ increased $<10 \%$ after fluid loading. SVI, stroke volume index.

Table 2 Hemodynamic changes of responders and non-responders before and after VE

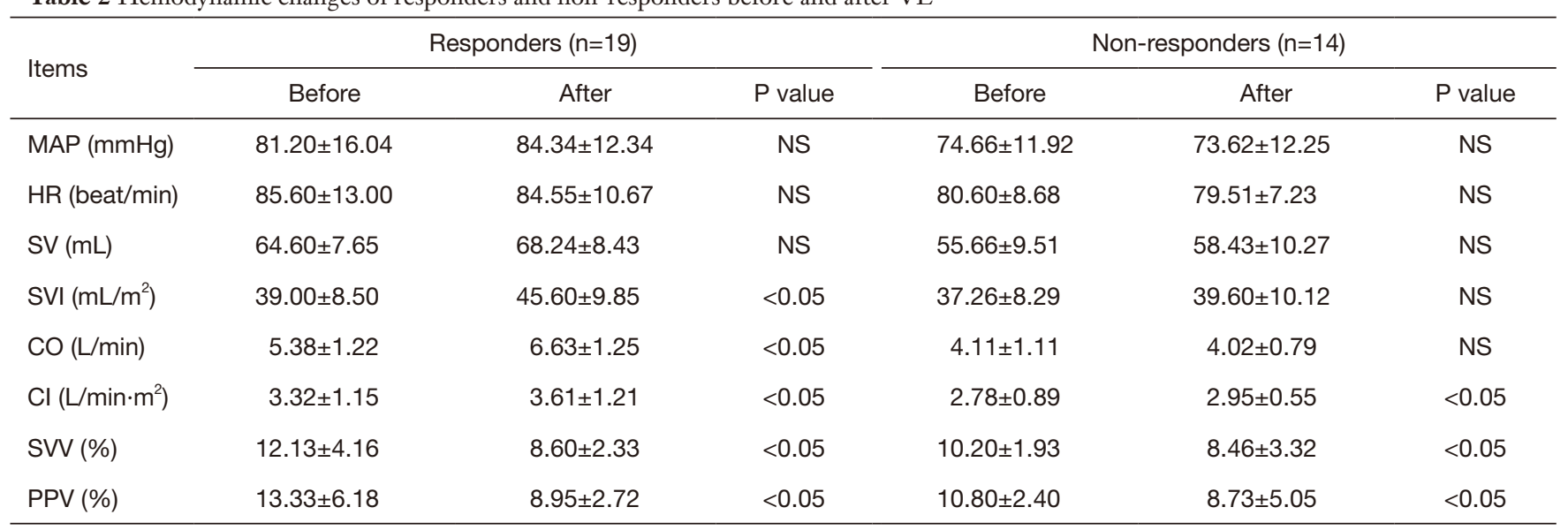

Values are mean $\pm \mathrm{SD}$. VE, volume expansion; MAP, mean arterial pressure; HR, heart rate; SV, stroke volume; SVI, stroke volume index; $\mathrm{CO}$, cardiac output; $\mathrm{Cl}$, cardiac output index; SVV, stroke volume variation; PPV, pulse pressure variation; NS, not significant.

\section{Results}

\section{Patient selection}

A total of 35 patients were initially included in this study, 2 of which were excluded due to the frequent premature ventricular contractions during the study. The characteristics of the 33 studied patients are presented in Table 1. According to the results of hemodynamic changes, 19 patients were classified as responders and 14 patients were non-responders. There were no significant differences in patient characteristics between responders and nonresponders (Table 1).

\section{Hemodynamic changes}

Table 2 shows the changes in hemodynamic indexes before and after VE between the two groups. After VE, there were no significant changes in HR, MAP, and SV in both responders and non-responders, but CI were significantly changed in the two groups. SVI and CO increased significantly in responders before and after $\mathrm{VE}$, but not in non-responders. VE also caused decreases in PPV and SVV in both responders and non-responders.

\section{Correlation analysis of SVV, PPV, and $\triangle S V I$ before $V E$}

Before VE, the SVV and PPV correlated with $\triangle$ SVI in responders $(\mathrm{r}=0.621, \mathrm{r}=0.569$, respectively, $\mathrm{P}<0.05$, Figure 2$)$, but neither the SVV nor PPV correlated with $\triangle \mathrm{SVI}$ in nonresponders $(\mathrm{P}>0.05)$.

\section{Dynamic indices to predict fluid responsiveness}

The overall performance of SVV and PPV in predicting the 
A
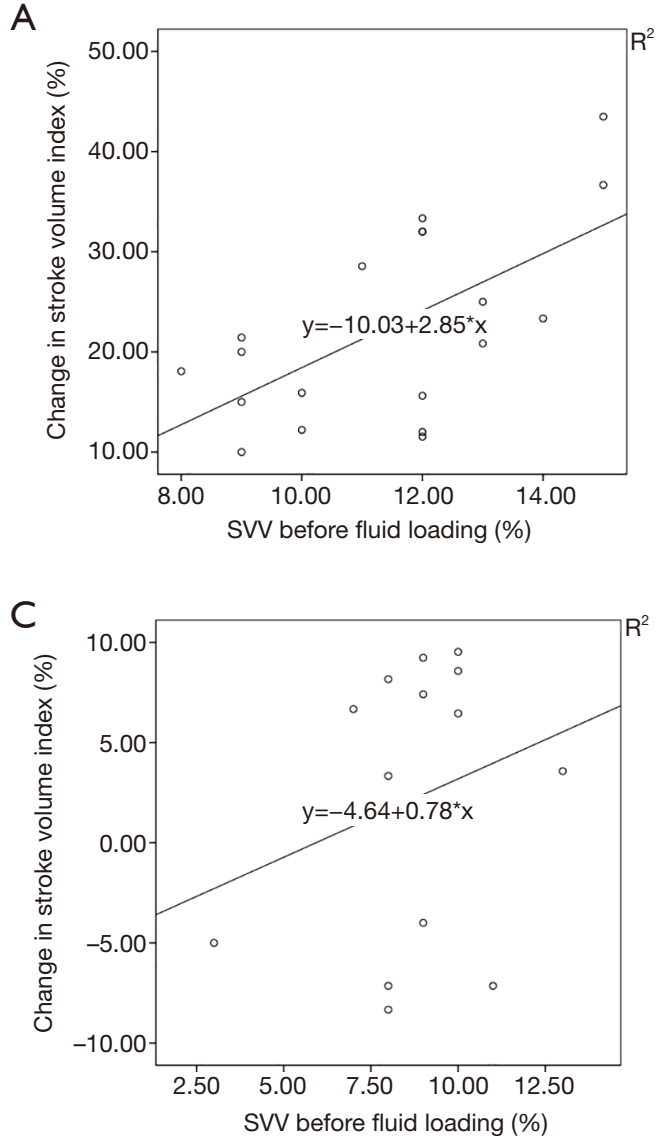

B

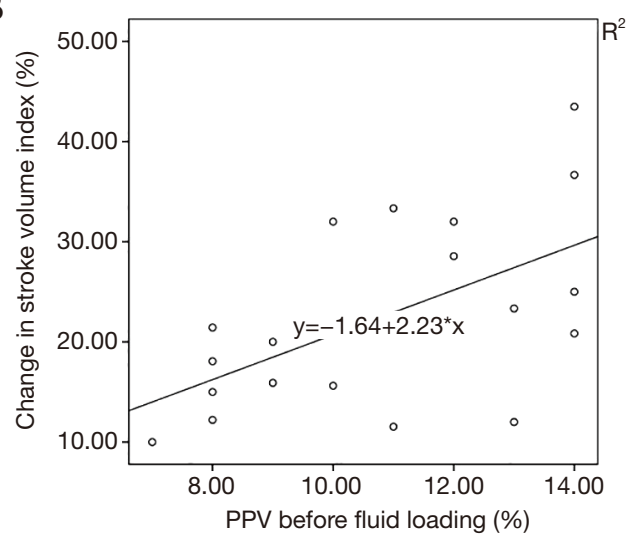

$\mathrm{D}$

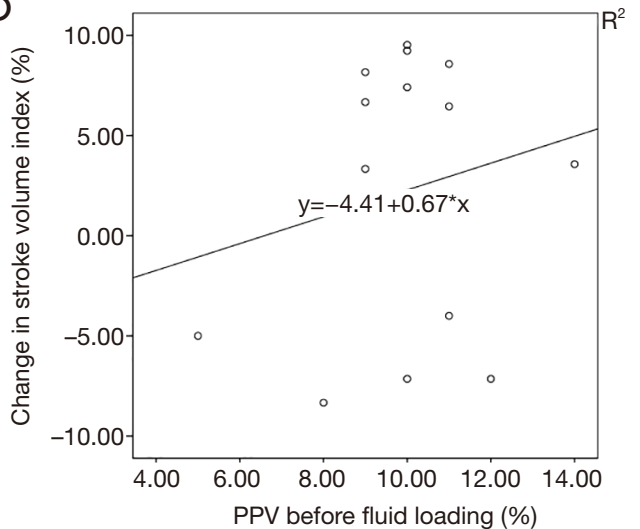

Figure 2 Correlations between the SVV, PPV, and $\triangle$ SVI before VE in responders and non-responders. Before VE, the SVV and PPV correlated with the $\Delta \mathrm{SVI}$ in responders [(A) $\mathrm{r}=0.621, \mathrm{P}<0.01$; (B) $\mathrm{r}=0.569, \mathrm{P}<0.05$ ]. However, there were no significant correlations between SVV, PPV, and $\triangle$ SVI in non-responders [(C) $\mathrm{r}=0.225, \mathrm{P}>0.05$; (D), $\mathrm{r}=0.199, \mathrm{P}>0.05]$. SVV, stroke volume variation; PPV, pulse pressure variation; SVI, stroke volume index; VE, volume expansion.

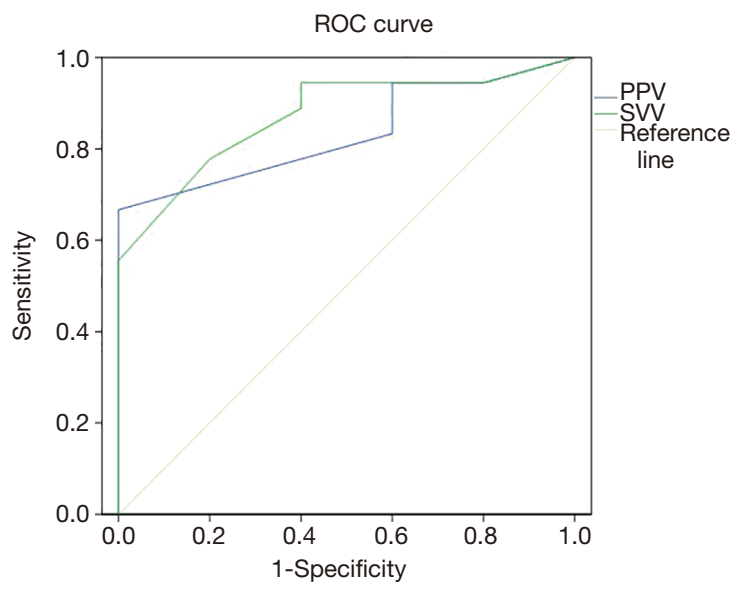

Figure 3 ROC curve of SVV and PPV to predict fluid responsiveness in the two groups. ROC, receiver operating characteristic; SVV, stroke volume variation; $\mathrm{PPV}$, pulse pressure variation. responsiveness of $\mathrm{SV}$ to intravascular $\mathrm{VE}$ was evaluated by constructing ROC curves (Figure 3). The areas under the ROC curves of patients with thoracic kyphosis were 0.872 (95\% CI: $0.719-1.000)$ for SVV and 0.833 (95\% CI: $0.667-$ 1.000) for PPV. The threshold for the SVV of patients with thoracic kyphosis was $13.5 \%$, and the threshold for the PPV was $14.5 \%$. The ROC curves showed that both SVV and PPV could accurately predict fluid responsiveness in patients with thoracic kyphosis. There was no significant difference between the areas under the ROC curves of SVV and PPV $(\mathrm{P}<0.05)$.

\section{Discussion}

SVV and PPV are currently important functional hemodynamic parameters in volume management, the trends 
of which can be used to evaluate the ability to change the volume load of SV and CO $(8,9)$. However, many factors can influence their accuracy, such as body position, abdominal pressure, tidal volume, and vasoactive drugs (10-13).

Kyphosis is a pathological spinal deformity, usually secondary to ankylosing spondylitis, spinal trauma, tuberculosis, and other diseases, and is due to pathological changes resulting in muscle ligament relaxation, osteomalacia, and bone deformity caused by the role of gravity. Studies have shown that when the Cobb's angle is more than 60 degrees, the deformity will continue to be aggravated, leading to back pain or even paraplegia, which generally requires corrective treatment. Multiple thoracolumbar vertebrae are often involved in kyphosis surgery. During the operation, the exposure of the vertebral body, the placement of pedicle screws, and the resection of hemivertebra will cause a large amount of blood loss, and the blood loss of some patients can reach tens of thousands of $\mathrm{ml}$. In order to maintain the stability of perioperative circulation and the perfusion of organs, anesthesiologists need to input a large number of fluids, including crystalloid fluids and various kinds of colloid fluids, while importing blood products. Therefore, an index is needed that can accurately reflect the patient's volume in kyphosis corrective surgery.

In patients with thoracic kyphosis, respiratory resistance increases due to thoracic deformity and poor chest wall compliance (14). Patients with scoliosis often develop this condition due to a congenital reason, while patients with kyphosis often develop this condition secondary to ankylosing spondylitis. It was reported that in severe cases of thoracic kyphosis, not only respiratory function was limited, but small pulmonary airways and the capillary bed could be distorted, which increased respiratory circulation resistance and made patients prone to complications such as pneumonia, atelectasis, and respiratory insufficiency $(15,16)$. Therefore, will the pathophysiology of thoracic kyphosis affect the accuracy and thresholds of SVV and PVV to predict fluid responsiveness? A clear conclusion has been lacking until now. Biais et al. showed that PPV and SVV in the prone position were lower than in the supine position during the scoliosis operation (17). However, no one has explained how thoracic deformity and scoliosis affect SVV and PPV, and there have been no studies on how SVV and PPV work in patients with thoracic kyphosis. In addition, patients with thoracic kyphosis are often frail, with decreased pulmonary compliance and cardiac reserve function, and have poor adaptability to fluid responsiveness (18).

Our study shows that both SVV and PPV can be used to predict fluid responsiveness in patients with thoracic kyphosis. The baseline values of SVV and PPV were linearly correlated with $\Delta$ SVI, so the higher baseline values of patients with thoracic kyphosis before VE were, the higher the predictive value was. The areas under the ROC curves showed the ability of the hemodynamic parameters to discriminate between responders and non-responders after VE. In our study, we found a threshold value of $13.5 \%$ for SVV and $14.5 \%$ for PPV in patients with thoracic kyphosis. The threshold values of thoracic kyphosis were both higher to normal (13\% for SVV and $13.5 \%$ for PPV). This may be due to the decrease of chest volume and chest wall compliance in patients with kyphosis, which may affect the accuracy and thresholds of SVV and PPV in predicting volume response. When the abdominal and thoracic pressure increases, the volume of blood in the veins and the resistance of the whole body are increased, and the $\mathrm{SV}$ and $\mathrm{CO}$ are decreased. According to the formula $\mathrm{SVV}=\left(\mathrm{SV}_{\text {max }}-\mathrm{SV}_{\text {min }}\right) / \mathrm{SV}_{\text {mean }}$, intrapleural pressure increases and vena cava reflux decreases during the whole anesthesia process, which results in decreases in the $\mathrm{SV}_{\text {mean }}$ value during the whole positive pressure ventilation process. As the difference of $\mathrm{SV}_{\max }-\mathrm{SV}_{\min }$ remains unchanged, $\mathrm{SVV}$ will increase. In the same way, the threshold of PPV will also increase.

Especially note that, mechanical ventilation is a necessary condition according to the principle of SVV. The accuracy of SVV is very poor under the condition of spontaneous breathing, because the tidal volume of spontaneous breathing changes greatly, which will artificially increase the change of SV with breathing (19). PPV is the same as SVV.

There are some limitations in our research. Firstly, all patients had thoracic kyphosis, and it remains unclear whether lesions involving abdomen kyphosis may influence the accuracy of SVV and PPV to predict fluid responsiveness. Secondly, we recorded hemodynamic data before the operation in the prone position, and we do not know whether it will be the same in the supine position. Thirdly, the patients we chose all had normal heart function (LVEF >50\%), while some severe patients with poor heart and lung function were not selected. Therefore, we cannot infer whether PPV or SVV can be used in severe patients with thoracic kyphosis. Fourthly, we only used the FloTrac system for measurement. In the future, thermodilution and echo technology can be used to further prove the effectiveness of hemodynamic parameters. Lastly, the 
sample size of our study was relatively small, and research with larger sample sizes would provide more accurate results.

In conclusion, both SVV and PPV can accurately predict fluid responsiveness in patients with thoracic kyphosis during the operation. However, we should pay more attention to the changes in thresholds in clinical work.

\section{Acknowledgments}

We thank Professor Heather B. Wallace (USA) and Professor Jiali Cai (Jiangnan University, China) for proofreading the article.

Funding: Innovation and cultivation fund of the sixth medical center of PLA General Hospital (No. CXPY201733).

\section{Footnote}

Reporting Checklist: The authors have completed the STARD reporting checklist. Available at https://dx.doi. org/10.21037/apm-21-1211

Data Sharing Statement: Available at https://dx.doi. org/10.21037/apm-21-1211

Conflicts of Interest: All authors have completed the ICMJE uniform disclosure form (available at https://dx.doi. org/10.21037/apm-21-1211). The authors have no conflicts of interest to declare.

Ethical Statement: The authors are accountable for all aspects of the work in ensuring that questions related to the accuracy or integrity of any part of the work are appropriately investigated and resolved. This study followed the tenets of the Declaration of Helsinki (as revised in 2013) and was approved by the Ethics Committee of the Chinese PLA general hospital and written informed consent was obtained from each participant after the nature and possible results of the study were explained (Trial registration number: S2020-006-02).

Open Access Statement: This is an Open Access article distributed in accordance with the Creative Commons Attribution-NonCommercial-NoDerivs 4.0 International License (CC BY-NC-ND 4.0), which permits the noncommercial replication and distribution of the article with the strict proviso that no changes or edits are made and the original work is properly cited (including links to both the formal publication through the relevant DOI and the license). See: https://creativecommons.org/licenses/by-nc-nd/4.0/.

\section{References}

1. Li C, Yang M, Wang C, et al. Preoperative factors predicting intraoperative blood loss in female patients with adolescent idiopathic scoliosis. Medicine (Baltimore) 2015;94:e359.

2. Xu H, Zhang $\mathrm{Y}$, Zhao Y, et al. Radiologic and clinical outcomes comparison between single- and two-level pedicle subtraction osteotomies in correcting ankylosing spondylitis kyphosis. Spine J 2015;15:290-7.

3. Berkenstadt H, Margalit N, Hadani M, et al. Stroke volume variation as a predictor of fluid responsiveness in patients undergoing brain surgery. Anesth Analg 2001;92:984-9.

4. Kang WS, Kim JY, Woo NS, et al. The influence of different mechanical ventilator settings of peak inspiratory pressure on stroke volume variation in pediatric cardiac surgery patients. Korean J Anesthesiol 2014;66:358-63.

5. Hou JY, Zheng JL, Ma GG, et al. Evaluation of radial artery pulse pressure effects on detection of stroke volume changes after volume loading maneuvers in cardiac surgical patients. Ann Transl Med 2020;8:787.

6. Liu X, Fu Q, Mi W, et al. Pulse pressure variation and stroke volume variation predict fluid responsiveness in mechanically ventilated patients experiencing intraabdominal hypertension. Biosci Trends 2013;7:101-8.

7. Kim YK, Shin WJ, Song JG, et al. Comparison of stroke volume variations derived from radial and femoral arterial pressure waveforms during liver transplantation. Transplant Proc 2009;41:4220-8.

8. Cannesson M, Musard H, Desebbe O, et al. The ability of stroke volume variations obtained with Vigileo/FloTrac system to monitor fluid responsiveness in mechanically ventilated patients. Anesth Analg 2009;108:513-7.

9. $\quad \mathrm{Fu} \mathrm{Q}, \mathrm{Mi} \mathrm{WD}$, Zhang H. Stroke volume variation and pleth variability index to predict fluid responsiveness during resection of primary retroperitoneal tumors in Hans Chinese. Biosci Trends 2012;6:38-43.

10. Daihua Y, Wei C, Xude S, et al. The effect of body position changes on stroke volume variation in 66 mechanically ventilated patients with sepsis. J Crit Care 2012;27:416.e7-12.

11. Suehiro K, Okutani R. Influence of tidal volume for stroke volume variation to predict fluid responsiveness 
in patients undergoing one-lung ventilation. J Anesth 2011;25:777-80.

12. van Lavieren $M$, Veelenturf J, Hofhuizen C, et al. Dynamic preload indicators decrease when the abdomen is opened. BMC Anesthesiol 2014;14:90.

13. Wajima $Z$, Shiga T, Imanaga K, et al. Do induced hypertension and hypotension affect stroke volume variation in man? J Clin Anesth 2012;24:207-11.

14. Seidi F, Rajabi R, Ebrahimi I, et al. The efficiency of corrective exercise interventions on thoracic hyper-kyphosis angle. J Back Musculoskelet Rehabil 2014;27:7-16.

15. Koguchi-Yoshioka H, Wataya-Kaneda M, Nakano H, et al. Severe scoliosis associated with the WNT10A mutation. J Dermatol 2015;42:322-3.

16. Bamps S, Calenbergh FV, Loon JV, et al. Posterolateral approach in a neurofibromatosis type-I patient with severe

Cite this article as: Chen Y, Guo X, Fu J, Dong T, Liu X, $\mathrm{Lv} \mathrm{H}$. Accuracy of stroke volume variation and pulse pressure variation to predict fluid responsiveness in patients with thoracic kyphosis. Ann Palliat Med 2021;10(7):7571-7578. doi: 10.21037/apm-21-1211 dystrophic thoracic kyphoscoliosis: a case report, cadaver study, and literature review. J Neurosci Rural Pract 2015;6:84-6.

17. Biais $\mathrm{M}$, Bernard $\mathrm{O}, \mathrm{Ha} \mathrm{JC}$, et al. Abilities of pulse pressure variations and stroke volume variations to predict fluid responsiveness in prone position during scoliosis surgery. Br J Anaesth 2010;104:407-13.

18. Zhang H, Guo C, Tang M, et al. Prevalence of scoliosis among primary and middle school students in Mainland China: a systematic review and meta-analysis. Spine (Phila Pa 1976) 2015;40:41-9.

19. Pinsky MR. Functional hemodynamic monitoring. Crit Care Clin 2015;31:89-111.

(English Language Editor: C. Betlazar-Maseh) 